\title{
AVALIAÇÃO DOS IMPACTOS DA COVID-19 NA MORTALIDADE POR SÍNDROME RESPIRATÓRIA AGUDA GRAVE NO ESTADO DO RIO GRANDE DO SUL, BRASIL ${ }^{1}$
}

\author{
EVALUATION OF THE IMPACTS OF COVID-19 ON MORTALITY FROM \\ SEVERE ACUTE RESPIRATORY SYNDROME IN THE STATE OF \\ RIO GRANDE DO SUL, BRAZIL
}

\section{Rivaldo Faria ${ }^{2}$, Maurício Rizzatti ${ }^{3}$, Natália Lampert Batista ${ }^{4}$, Pedro Leonardo Cezar Spode ${ }^{5}$ e Douglas Bouvier Erthal ${ }^{6}$}

\section{RESUMO}

Desde a chegada da COVID-19, o Brasil vive uma explosão de internações e óbitos por Síndrome Respiratória Aguda Grave (SRAG), em valores acima da série histórica. Existe a preocupação de que a COVID-19 possa estar sendo registrada no sistema de controle da SRAG, mas sem a classificação etiológica. O objetivo deste estudo é analisar os impactos da COVID-19 no aumento da SRAG no Rio Grande do Sul, considerando-se às dimensões demográfica, racial, escolaridade e de privação social. As taxas de mortalidade foram estimadas através do método bayesiano empírico e mapeadas em Sistema de Informação Geográfica. A privação social foi avaliada através de um modelo multicritério de indicadores de renda, educação e saúde. Os resultados mostraram aumento da mortalidade por SRAG e variações demográficas condizentes com os impactos da COVID-19. Observou-se ainda o elevado número de óbitos sem a identificação etiológica, que sugere a necessidade urgente de aumentar os testes de COVID-19 no estado. A maior concentração dos óbitos ocorreu no eixo mais desenvolvido e de menor privação social do estado, mas as taxas são maiores entre negros, indígenas e na população de baixa escolaridade. Os casos se estendem em quase todas as regiões do estado e revelam preocupação em relação aos municípios de elevada privação social nas fronteiras sul e oeste. Recomenda-se, pois, o desenvolvimento de ações adequadas às populações mais vulneráveis, o aumento dos testes e políticas de prevenção para o estado que possui o inverno mais rigoroso e a população proporcionalmente mais idosa do país.

Palavras-chave: SARS-CoV-2, Síndrome Respiratória Aguda Grave, Coronavírus, análise espacial.

\section{ABSTRACT}

Since the beginning of COVID-19, Brazil has experienced an explosion of hospitalizations and deaths due to Severe Acute Respiratory Syndrome (SARS), in values above the historical series. There is concern that COVID-19 may have been registered in the SARS control system, but without etiological classification. The objective of this study is to analyze the impacts of COVID-19 on the increase of SARS in Rio Grande do Sul, considering the demographic, racial, school and social private dimensions. Mortality rates were estimated using the empirical bayesian method and mapped using the Geographic Information System. Social deprivation was assessed using a multicriteria model that includes income, education and health indicators. The results

\footnotetext{
${ }^{1}$ Trabalho de Pesquisa.

${ }^{2}$ Prof. Dr. do Departamento de Geociências e Programa de Pós-Graduação em Geografia - Universidade Federal de Santa Maria. E-mail: rivaldogeo@hotmail.com

${ }^{3}$ Doutorando em Geografia - Universidade Federal de Santa Maria. E-mail: geo.mauricio.rizzatti@gmail.com

${ }^{4}$ Pós-doutoranda em Geografia - Universidade Federal de Santa Maria. E-mail: natilbatista3@gmail.com

${ }^{5}$ Doutorando em Geografia - Universidade Federal de Santa Maria. E-mail: pedrospode@gmail.com

${ }^{6}$ Mestrando em Geografia - Universidade Federal de Santa Maria. E-mail: douglasbouv@gmail.com
} 
showed increased mortality from SARS in 2020 and changed demographic conditions with the impacts of COVID-19. Also note the high number of deaths without etiological identification, which suggests an urgent and immediate need to increase COVID-19 tests for all deaths from SARS. The highest concentration of deaths occurred in the most developed and least socially deprived municipalities, but the rates are higher among blacks, indigenous people and the population with low education. The cases extend in almost all regions of the state and lead to concern in relation to municipalities with high social deprivation. It is recommended, therefore, the development of appropriate actions for the most vulnerable populations, the increase of tests and prevention policies for the state that has the most severe winter and the proportionally older population in the country.

Keywords: SARS-CoV-2, Severe Acute Respiratory Syndrome, Spatial Analysis.

\section{INTRODUÇÃO}

A COVID-19 já é a maior crise de saúde pública do mundo e o Brasil é hoje um dos seus principais epicentros (LOBO et al., 2020). Todo evento epidêmico novo, traz inúmeros desafios para as ações de vigilância e as respostas e impactos são, quase sempre, muito diferentes entre países e regiões. Um dos maiores desafios é ter a real dimensão da epidemia, que vai desde a compreensão das características físico-química-biológica do vírus, até o estudo da dinâmica da sua transmissão e propagação. Pelos aprendizados com a SARS-CoV, em 2003, e a MERS-CoV. em 2012, a China e demais países começaram a adotar medidas de isolamento social, que é, por enquanto, a melhor forma de conter sua propagação. Entretanto, a COVID-19 traz um desafio maior em relação aos coronavírus anteriores, que é sua maior transmissibilidade, avaliada pelo número básico de reprodução $\left(\mathrm{R}_{0}\right)$ (LIU et al., 2020; LANA et al., 2020; CAO et al., 2020). A razão dessa maior propagação é geralmente associada pelo tempo entre incubação e os sintomas (entre 2,9 e 4,2 dias), como também ao elevado número de casos assintomáticos que podem transmitir o vírus (MIZUMOTO, 2020).

A rápida propagação do vírus e sua difusão espacial produz um cenário de incerteza quanto à real dimensão da epidemia e seus impactos na saúde da população. Por isso, a recomendação é aumentar a cobertura de testes, como o fizeram vários países desenvolvidos (LEE et al., 2020; CHEN et al., 2020; MARCEL et al., 2020). Essa é a forma mais eficaz para dimensionar as ações da vigilância, seja para recomendar ações mais flexíveis ou mais severas de isolamento social, seja ainda para direcionar os próprios recursos humanos e tecnológicos. Do contrário, será um planejamento de remediação, sempre num campo cego, avaliado apenas em relação aos testes realizados com os casos sintomáticos e hospitalizados.

Entretanto, é um desafio enorme para o Brasil aumentar a cobertura de testes para COVID-19, seja pela sua extensão geográfica, sua enorme população e, principalmente, a falta de um planejamento integrado entre os entes federativos. Estudos mostram que as notificações representam apenas cerca de 8\% dos casos (PRADO et al., 2020; HALLAL et al., 2020; UFPEL, 2020). Portanto, há um enorme subdimensionamento da enfermidade, como também, pelo menos por enquanto, pelos óbitos 
por ela representados. Uma forma de avaliar a subnotificação, aliás, é medir a série histórica dos óbitos e comparar com o mesmo período da epidemia. Mas ainda não há dados atualizados e disponíveis dos óbitos para o ano de 2020.

Um indicador importante que pode ser usado para avaliar os impactos da COVID-19 são os casos hospitalizados por Síndrome Respiratória Aguda Grave (SRAG). Desde 2009 a hospitalização por SRAG é registrada no Sistema Nacional de Agravos de Notificação (SINAN), e incluía, até 2019, os vírus da influenza A e B (H1N1), sincicial respiratório (VSR), Adenovírus e Parainfluenza (BASTOS et al., 2020). Neste ano de 2020 o teste para a SARS-CoV-2 foi incluído no protocolo. Alguns estudos já mostram o aumento das internações a partir do mês de março deste ano, em valores bem superiores à série histórica, o que pode indicar que os casos graves de COVID-19 estejam sendo a detectados pelo sistema de registro das SRAG (BASTOS et al., 2020; ALONSO et al. 2020; FIOCRUZ, 2020; LISBOA et al., 2015). Todavia, trata-se de estudos com dados globais, para todo o país, e sem a avaliação das variações espaciais, que é algo importante num país da dimensão do Brasil. Além disso, são estudos baseados nos resultados gerais das internações, faltando ainda investigações focadas nos óbitos, que são dados mais objetivos para compreender o possível impacto da COVID-19 nos registros de SRAG.

Inúmeras razões podem levar a variações espaciais na mortalidade por SRAG, por isso, é recomendado avaliar cada região considerando-se suas características sociais e demográficas, como também suas peculiaridades físicas e geográficas locais. O Rio Grande do Sul, por exemplo, dada suas características territoriais (clima, longa fronteira, economia, etc.), pode apresentar situações variadas de internação e óbitos por SRAG. O mais recente Boletim Infogripe da Fiocruz (2020) avaliou o estado como zona de risco, com forte tendência ao aumento dos casos no inverno. Já é reconhecida a maior sobrecarga de internações por SRAG no inverno gaúcho, mas a difusão da COVID-19 e a falta de exames para a rápida detecção e controle, podem levar não apenas a subnotificação, mas ao colapso do sistema de saúde. Considerando esse contexto, o primeiro objetivo deste trabalho é dimensionar a frequência e a taxa de mortalidade por SRAG entre março e junho de 2020 no Rio Grande do Sul, tendo como referência comparativa os cinco anos anteriores. O segundo objetivo propõe avaliar a evolução da mortalidade por SRAG considerando-se as variáveis demográficas, de raça e escolaridade no mesmo período. O terceiro objetivo, finalmente, pretende compreender a dinâmica espacial da mortalidade por SRAG e COVID-19 no mesmo período, tendo em vista as condições sociais avaliadas a partir de um indicador multicritério de privação social para os municípios do Rio Grande do Sul.

\section{MATERIAL E MÉTODO}

Este é um estudo de base populacional realizado com todos os óbitos por SRAG (incluindo-se a COVID-19) registrados nos meses de março a junho de 2015 a 2020. Trata-se de uma análise histórica univariada, em intervalo definido a partir da avaliação da variação do histograma de toda série 
histórica no sistema Infogripe (FIOCRUZ, 2020). O período de cinco anos foi considerado consistente para a verificação da proposição do aumento de mortes por SRAG no mesmo período no ano de 2020. O estudo foi aplicado para os meses de março a junho por compreenderem a série mensal completa de registro de casos de COVID-19 no Rio Grande do Sul. Não foi avaliada a sazonalidade do evento, por considerar que para isso é necessário ter dados de toda a série e de pelo menos duas estações completas, nem os dados do mês de julho, pois o mês ainda não se completou.

Todos os dados foram coletados no painel Coronavírus/Brasil (https://covid.saude.gov.br/) e modelados ao nível dos municípios do estado do Rio Grande do Sul. A taxa de mortalidade foi calculada em relação à população residente projetada pelo Instituto Brasileiro de Geografia e Estatística (IBGE) para os respectivos anos avaliados. Posteriormente, essa taxa bruta foi reestimada através do método bayesiano empírico, devido as oscilações aleatórias comuns em estudos de base populacionais envolvendo áreas. De acordo com Câmara et al. (CÂMARA et al., 2004), o bayesiano empírico parte do pressuposto de que a taxa $\theta_{i}$ é uma variável aleatória, que contém uma média $\mu_{i}$ e uma variância $\sigma_{1}^{2}$. Então, para calculá-lo realiza-se uma combinação entre a taxa observada $\left(t_{i}\right)$, a média $\left(\mu_{i}\right)$ e o peso de confiança $\left(w_{i}\right)$ do indicador para cada microrregião, conforme descrito na equação:

$$
\begin{gathered}
\text { O fator } w_{i} \text { é dado por: } \quad \theta_{i}=w_{i} t_{i}+\left(1-w_{i}\right) \mu_{i} \\
w_{i}=\frac{\sigma_{i}^{2}}{\sigma_{i}^{2}+u_{i} / n_{i}}
\end{gathered}
$$

A frequência dos óbitos, por seu turno, foi calculada em relação a proporção relativa de óbitos, que é o número de óbitos distribuídos pela população residente de cada grupo avaliado. Isso foi feito porque os grupos estudados (idade, raça e escolaridade) têm tamanhos populacionais muito diferentes e calcular a frequência relativa apenas pelo número total de óbitos, como comumente é feita, levaria a erros de se comparar a frequência em relação dimensões amostrais diferentes.

Para avaliar as condições sociais dos territórios foi elaborado um indicador de avaliação multicritério, assim chamado Índice de Privação Social (IPS), conforme orientação de Carstairs e Morris (1990). A construção é feita em quatro etapas que vão da seleção das variáveis, à padronização numa mesma escala de medida, ponderação e combinação dos critérios. Neste estudo foram selecionados seis indicadores que avaliam as dimensões da educação, renda e saúde da população, todos coletados do IBGE e do Atlas do Desenvolvimento Humano (PNUD, 2013). São eles: (i) percentual da renda apropriada pelos 40\% mais pobres; (ii) razão de dependência; (iii) esperança de vida ao nascer; (iv) taxa de analfabetismo da população de 15 anos ou mais; (v) percentual de vulneráveis à pobreza; (vi) percentual da população inativa com renda igual ou inferior a meio salário mínimo. A seleção foi feita em rodadas de avaliação seguindo os critérios da representatividade e fiabilidade dos indicadores. A padronização ou normalização foi feita pela variabilidade máxima e mínima de cada categoria, que é a mesma 
técnica usada na elaboração do Índice de Desenvolvimento Humano (IDH). A ponderação, por seu turno, foi operada com uso do Método de Análise Hierárquica (Analytic Hierarchy Process) (SAATY, 1990). Finalmente, a combinação dos critérios padronizados e ponderados, foi feita utilizando-se a técnica da combinação linear ponderada, que combina os fatores de acordo com a média ponderada (SAATY, 1990). O IPS final varia de 0 a 1 , sendo maior a privação quanto mais próximo de 1 e vice-versa.

As análises foram realizadas com uso das técnicas exploratórias de dados espaciais, em Sistema de Informação Geográfica (SIG), através do programa ArcGis, da empresa Esri ${ }^{\circledR}$, onde foram elaborados os mapas coropléticos usados no trabalho. Para tornar possível a comparação dos mapas das taxas de mortalidade em séries temporais, a classificação foi feita manualmente em cinco classes divididas em intervalos regulares. A definição dos cortes das classes foi feita considerando-se o intervalo regular de distância entre a média geral e o menor valor do período ( $1^{\mathrm{a}}$ corte), a própria média geral ( $2^{\circ}$ corte), a média da taxa para o ano de 2020 ( $3^{\circ}$ corte $)$ e o intervalo igual de distância entre a média do ano de 2020 e o valor máximo do período avaliado ( $4^{\circ}$ corte). Como os casos são muito maiores no ano de 2020, a média da taxa calculada e o valor máximo deste ano foram tomados como referência para os últimos cortes de classe. Isso foi necessário para garantir que toda a série histórica pudesse ser devidamente representada no mapa.

Para o mapa da densidade de COVID-19 e SRAG por municípios, utilizou-se o "Kernel Density", do programa ArcGis, que consiste em uma forma de quantificar as relações dos pontos dentro de um raio $(\mathrm{R})$ de influência, com base em determinada função estatística, analisando os padrões traçados por determinado conjunto de dados pontuais, estimando sua densidade na área de estudo (RIZZATTI et al., 2020). Dessa forma, o valor do raio adotado foi de $120 \mathrm{~km}$, pois se mostrou adequado para não gerar descontinuidades na interpolação e nem mascarar as classes todas em um maior valor.

\section{RESULTADOS}

Entre 2015 e 2020 foram registrados 2.917 óbitos por SRAG entre os meses de março a junho no estado do Rio Grande do Sul. O ano de 2020 registrou o maior número de casos (2.042), equivalente a 70\% do total. Destes, 617 foram classificados etiologicamente como COVID-19, apenas 4 por influenza e 9 por outro agente etiológico. Os demais (1.412) não tiveram razão etiológica classificada (Tabela 1). O ano de 2016, apontado como o maior pico da epidemia de H1N1 desde 2009 (CEVS, 2020), registrou 430 óbitos. Destes, 192 tiveram classificação para o vírus influenza, 8 por outro vírus respiratório, 2 por outro agente etiológico e 228 não foram especificados. Nos demais anos a frequência absoluta variou entre 97 e 129 óbitos, respectivamente nos anos de 2018 e 2019.

Em todos os anos, é elevado o número de óbitos não especificados, ou seja, sem a avaliação da causa etiológica da SRAG, uma média de 67,4\%. Das classificações especificadas, a influenza foi a 
principal causa dos óbitos entre 2015 e 2019. O maior número de óbitos classificados como influenza ocorreu no ano de 2016. No ano de 2020, a COVID-19 foi a principal causa de óbito com classificação etiológica (617 casos ou 30,2\% do total). A influenza foi identificada como razão para 4 óbitos e SRAG por outro agente etiológico foi a classificação para 9 óbitos. Excetuando-se os óbitos os classificados como COVID-19, o ano de 2020 registrou 1.425, o que equivale a mais que a soma total de todos os casos de óbitos por SRAG registrados nos anos de 2015 a 2016 (um total de 875).

Tabela 1 - Frequência absoluta e classificação dos óbitos por Síndrome Respiratória Aguda Grave, entre os meses de março e junho de 2015 a 2020, Rio Grande do Sul, Brasil.

\begin{tabular}{lcccccc}
\hline \multicolumn{1}{c}{ Classificação } & $\mathbf{2 0 1 5}$ & $\mathbf{2 0 1 6}$ & $\mathbf{2 0 1 7}$ & $\mathbf{2 0 1 8}$ & $\mathbf{2 0 1 9}$ & $\mathbf{2 0 2 0}$ \\
\hline SRAG por influenza & 11 & 192 & 31 & 27 & 18 & 4 \\
\hline SRAG por outro vírus respiratório & 3 & 8 & 5 & 3 & 14 & 0 \\
SRAG por outro agente Etiológico & 3 & 2 & 4 & 0 & 0 & 9 \\
COVID-19 & 0 & 0 & 0 & 0 & 0 & 617 \\
SRAG não especificado & 90 & 228 & 72 & 67 & 97 & 1412 \\
Total de casos & 107 & 430 & 112 & 97 & 129 & 2042 \\
\hline
\end{tabular}

Fonte: SINAN e SIVEP-Gripe.

A frequência relativa de casos foi maior entre a população de 60 anos ou mais em todos os anos avaliados (Tabela 2). Essa frequência variou entre 33,8\% e 49,3\% nos anos de 2015 a 2019 e alcançou 70,2\% no ano de 2020. Nos anos de 2015, 2016 e 2017 essa frequência foi alta também nas faixas etárias de 5 a 9 anos e 50 a 59 anos. No ano de 2018 também foram altas as frequências relativas de óbitos na população de 0 a 4 anos e 50 a 59 anos. O ano de 2020 diferencia-se dos demais pela frequência relativa muito mais acentuada na população idosa e pela baixa frequência relativa na população até 39 anos, sobretudo nas faixas etárias de 5 a 29 anos.

A taxa de mortalidade por SRAG, calculada por $100 \mathrm{mil} /$ habitantes, foi maior nos anos de 2016 e 2020 (3,8 e 18,1, respectivamente). Nos demais anos, essa taxa variou entre 0,9 a 1,1. O número muito maior de óbitos no ano de 2020 se reflete numa taxa de mortalidade muito maior que os anos anteriores, sobretudo entre os idosos. A média da mortalidade por SRAG na população idosa entre 2015 e 2019 foi de 4,7 por 100 mil/habitantes (variando de 2,3, em 2018, a 11,7, em 2016). No ano de 2020 essa taxa foi de 81,0. A taxa média de mortalidade para a faixa etária entre 0 e 9 anos foi de 1,2 entre 2015 e 2019 e 1,5 no ano de 2020, mostrando pouca diferença, sendo o menor valor registrado em 2015, na faixa entre 0 e 4 anos, e o maior valor em 2016, na faixa entre 5 e 9 anos. As maiores variações encontradas no ano de 2020, comparativamente aos anos anteriores, ocorreram nas idades acima dos 40 anos, sobretudo a população idosa, com taxas muito mais elevadas no corrente ano. 
Tabela 2 - Frequência relativa e taxa de mortalidade por Síndrome Respiratória Aguda Grave (SRAG) entre os meses de março a junho de 2015 a 2020, Rio Grande do Sul, Brasil.

\begin{tabular}{ccccccccccccc}
\hline \multicolumn{1}{c}{ Frequência relativa } & \multicolumn{4}{c}{ Taxa de mortalidade $^{1}$} \\
\hline Idade & $\mathbf{2 0 1 5}$ & $\mathbf{2 0 1 6}$ & $\mathbf{2 0 1 7}$ & $\mathbf{2 0 1 8}$ & $\mathbf{2 0 1 9}$ & $\mathbf{2 0 2 0}$ & $\mathbf{2 0 1 5}$ & $\mathbf{2 0 1 6}$ & $\mathbf{2 0 1 7}$ & $\mathbf{2 0 1 8}$ & $\mathbf{2 0 1 9}$ & $\mathbf{2 0 2 0}$ \\
\hline $0-4$ & 2,1 & 1,5 & 7,2 & 12,8 & 22,9 & 2,4 & 0,1 & 0,4 & 0,6 & 0,8 & 2,1 & 2,8 \\
\hline $5-9$ & 15,5 & 14,5 & 17,3 & 4,6 & 8,1 & 0,1 & 1,0 & 4,0 & 1,3 & 0,3 & 0,7 & 0,1 \\
$10-14$ & 0,0 & 0,5 & 1,8 & 6,3 & 1,5 & 1,2 & 0,0 & 0,1 & 0,1 & 0,4 & 0,1 & 1,4 \\
\hline $15-19$ & 0,0 & 1,7 & 4,6 & 1,8 & 0,0 & 0,7 & 0,0 & 0,5 & 0,4 & 0,1 & 0,0 & 0,8 \\
$20-29$ & 7,7 & 3,5 & 5,2 & 6,1 & 5,0 & 1,5 & 0,5 & 1,0 & 0,4 & 0,4 & 0,5 & 1,7 \\
\hline $30-39$ & 4,3 & 5,4 & 4,5 & 8,9 & 5,1 & 3,4 & 0,3 & 1,5 & 0,3 & 0,6 & 0,5 & 4,0 \\
$40-49$ & 9,9 & 12,0 & 7,7 & 6,1 & 5,8 & 7,2 & 0,7 & 3,4 & 0,6 & 0,4 & 0,5 & 8,2 \\
\hline $50-49$ & 11,1 & 18,9 & 13,1 & 17,7 & 17,8 & 13,3 & 0,8 & 5,3 & 1,0 & 1,2 & 1,6 & 15,3 \\
$\geq 60$ & 49,3 & 41,9 & 38,6 & 35,6 & 33,8 & 70,2 & 3,3 & 11,7 & 3,0 & 2,3 & 3,1 & 81,0 \\
\hline Total & 100 & 100 & 100 & 100 & 100 & 100 & 0,9 & 3,8 & 1,0 & 0,9 & 1,1 & 18,1 \\
\hline & \multicolumn{1}{c}{ Fonte: SINAN e SIVEP-Gripe. } & & & &
\end{tabular}

A taxa de mortalidade foi maior entre a população de cor preta nos anos de 2016 e 2020 e na população indígena ou amarela nos anos de 2015, 2017, 2018 e 2019 (Tabela 3). As menores taxas foram encontradas na população parda em todos os anos. Não foi encontrado valores no ano de 2020 que demostrasse diferenciação em relação aos anos anteriores, que é a tendência regular da maior taxa de mortalidade entre negros e indígenas.

A taxa de mortalidade é também maior na população com baixa escolaridade (Tabela 3). A população com ensino médio e ensino superior registrou as menores taxas em todos os anos avaliados. A diferença das taxas de mortalidade entre a população com instrução equivalente ou inferior ao ensino fundamental em relação a população com ensino médio e superior chega a uma média de quase 900\% entre os anos de 2015 a 2019. No ano de 2020 essa diferença foi um pouco menor, mas ainda alcançou uma média de mais de $400 \%$ de diferença.

Tabela 3 - Taxa de mortalidade por Síndrome Respiratória Aguda Grave (SRAG) por grupos de raça e grau de escolaridade, entre março e junho de 2015 a 2020, Rio Grande do Sul, Brasil.

\begin{tabular}{|c|c|c|c|c|c|c|c|}
\hline & Classificação & 2015 & 2016 & 2017 & 2018 & 2019 & 2020 \\
\hline \multirow{4}{*}{ 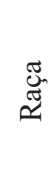 } & Branca & 0,9 & 3,6 & 1,0 & 0,9 & 1,1 & 15,7 \\
\hline & Preta & 1,3 & 4,5 & 0,5 & 1,0 & 1,3 & 18,6 \\
\hline & Indígena ou Amarela & 1,4 & 2,8 & 2,8 & 1,4 & 1,4 & 13,8 \\
\hline & Parda & 0,3 & 1,7 & 0,4 & 0,3 & 0,8 & 6,5 \\
\hline \multirow{5}{*}{ 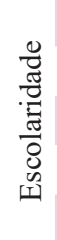 } & Analfabeto & 1,9 & 3,4 & 1,6 & 1,6 & 4,7 & 20,6 \\
\hline & Fundamental I & 0,6 & 2,7 & 0,9 & 0,8 & 0,7 & 9,1 \\
\hline & Fundamental II & 1,1 & 4,4 & 0,7 & 0,6 & 1,2 & 23,5 \\
\hline & Ensino Médio & 0,2 & 0,6 & 0,2 & 0,2 & 0,6 & 4,5 \\
\hline & Ensino Superior & 0,0 & 0,0 & 0,0 & 0,0 & 0,1 & 2,4 \\
\hline
\end{tabular}

Fonte: SINAN e SIVEP-Gripe

Cálculo da taxa por $100 \mathrm{mil} /$ habitantes. 
A distribuição espacial da taxa de mortalidade suavizada por municípios está representada na Figura 1. Excetuando-se o ano de 2016 e 2020, é pequena a extensão espacial do evento, com tendência à concentração entre a Região Metropolitana de Porto Alegre e Caxias do Sul, na Serra Gaúcha, e ao sul, entre Pelotas, Bagé e Uruguaiana. O ano de 2020 registrou óbitos em 279 municípios (56,1\%), onde está concentrada a maior parte da população do estado (91,0\%). Destes, 146 municípios registraram óbitos classificados etiologicamente como COVID-19. Todos os municípios que registraram óbitos por COVID-19 também registraram óbitos por SRAG não classificados como COVID-19. No ano de 2016, foram 132 municípios (26,6\%) com registro de óbitos por SRAG, onde vive 75,4\% da população do estado. Os demais anos registraram casos em menor número de municípios (entre 35 ou 7\%, em 2015, e 53 ou 10,1\%, em 2018), predominantemente nas médias e grandes cidades, razão da concentração populacional nestes municípios com registro de óbitos (entre 44,6\% e 53,5\% da população do estado, respectivamente nos anos de 2015 e 2017).

O cálculo da taxa suavizada é maior no ano de 2020, variando entre 5,6 e 49,1 por 100 mil/ habitantes. São elevadas as taxas nos municípios entre a Região Metropolitana e a Serra Gaúcha; entre Santa Maria e Bagé (no centro e sul); e entre Passo Fundo e Santa Rosa (no norte e noroeste). Comparativamente ao ano de 2020, os demais apresentaram menor variabilidade das taxas e valores da média igualmente muito mais baixos. Por isso, nos anos de 2015, 2017, 2018 e 2019, a maior parte dos municípios foi classificado no primeiro intervalo de classe do mapa (com taxa igual ou inferior a 3,6 por $100 \mathrm{mil} / \mathrm{hab}$.). Apenas o ano de 2016 alcançou maior número de municípios na segunda classe do mapa (até 9,6 óbitos por $100 \mathrm{mil} / \mathrm{hab}$.). O ano de 2020 não teve nenhum município classificado na primeira classe do mapa, sendo a maior parte deles com taxa acima de 9,6 por $100 \mathrm{mil} / \mathrm{hab}$.

Para além das taxas mais elevadas no ano de 2020, o evento apresenta-se geograficamente generalizado, presente em praticamente todas as regiões do estado.

Os mapas de densidade espacial das taxas suavizadas e do Índice de Privação Social (IPS) por municípios estão representados na Figura 2. A distribuição espacial das taxas de mortalidade (à direita) apresentou um comportamento regular em todos os anos, com maior densidade nos municípios localizados entre a região metropolitana de Porto Alegre (1), Caxias do Sul (2) e Passo Fundo (5), estendendo-se até o noroeste do estado, na região de Santa Rosa (8). Nos anos de 2016 e 2020 foram encontradas também elevadas densidades na região central, em Santa Maria (4), e ao sul, em Pelotas (3) e Bagé (7). As menores densidades foram encontradas na região do Pampa (sul e oeste). Embora o maior número de casos e as maiores taxas tenham ocorrido no ano de 2020, a distribuição espacial não difere do encontrado nos anos anteriores, que é densificação predominante no eixo capital-serra-norte do estado. 
Figura 1 - Densidade dos óbitos por Síndrome Respiratória Aguda Grave (à Direita) e Índice de Privação Social (IPS) (à esquerda), por municípios do Rio Grande do Sul, Brasil

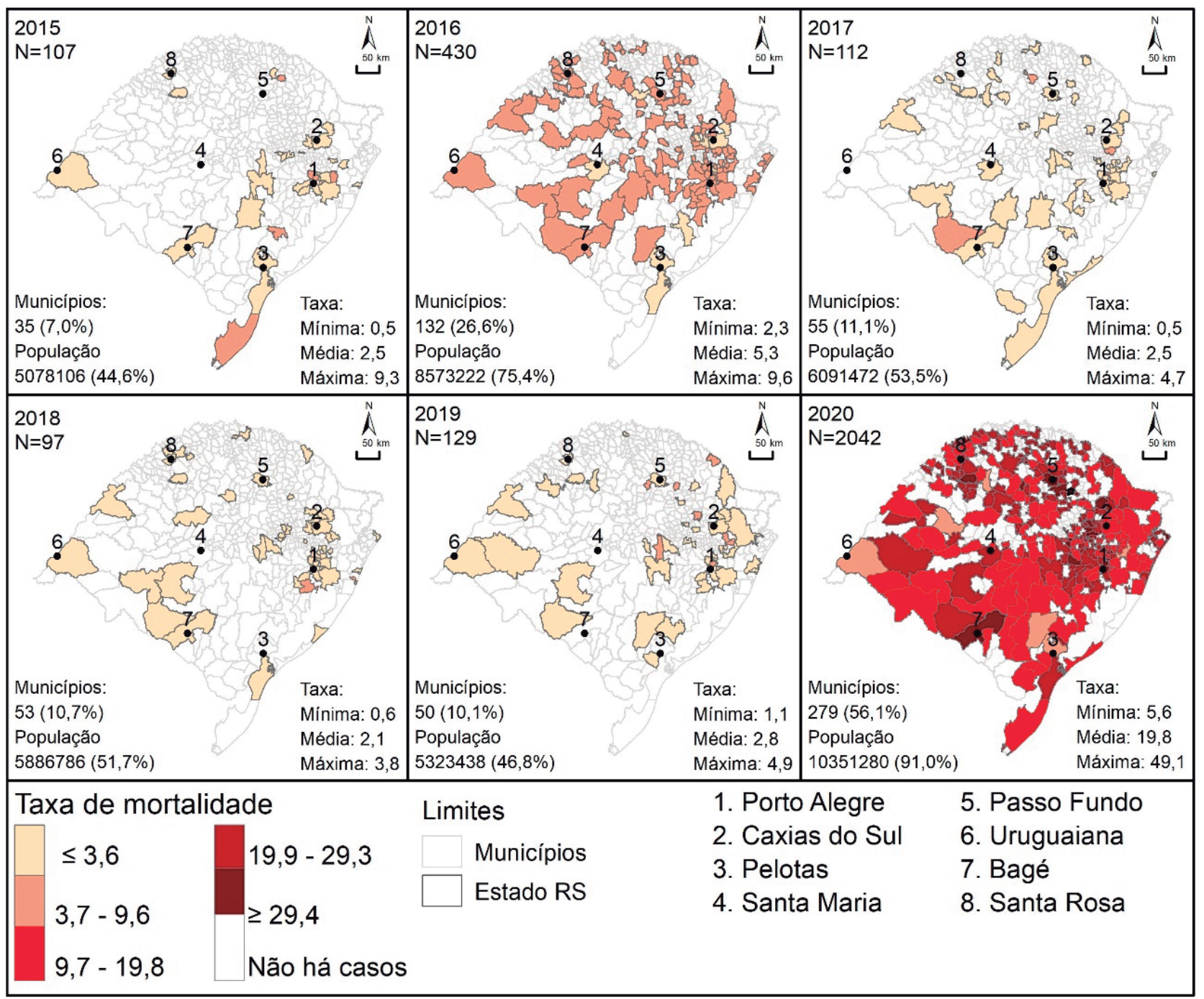


Figura 2 - Densidade dos óbitos por Síndrome Respiratória Aguda Grave (à Direita) e Índice de Privação Social (IPS) (à esquerda), por municípios do Rio Grande do Sul, Brasil.

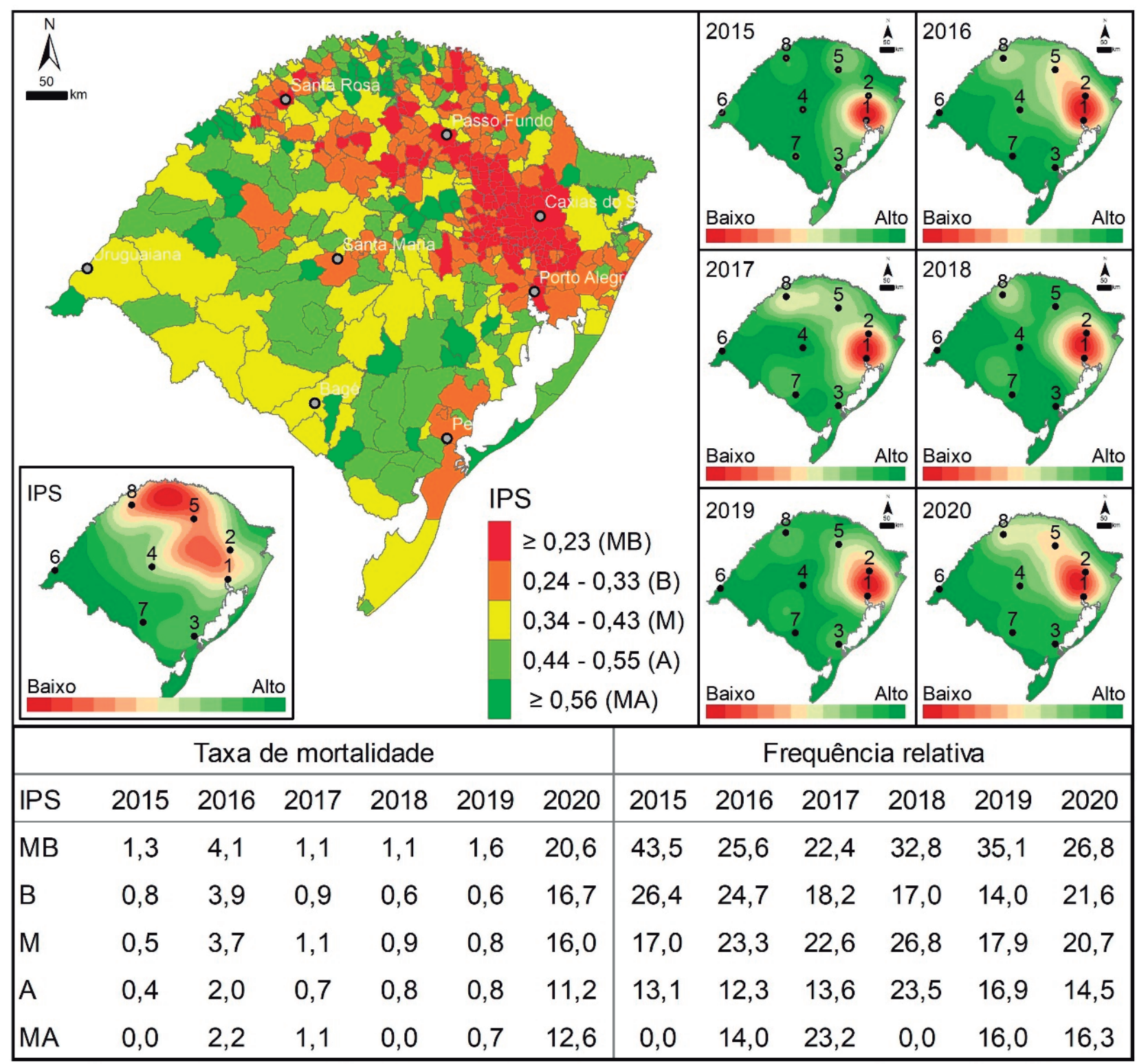

1. Porto Alegre; 2. Caxias do Sul; 3. Pelotas; 4. Santa Maria; 5. Passo Fundo; 6. Uruguaiana; 7. Bagé; 8. Santa Rosa

Os municípios com maiores IPS do Rio Grande do Sul estão localizados ao sul do estado, na região do Pampa, e no norte-nordeste, na divisa com Santa Catarina (Figura 2). Os municípios com menores IPS, por seu turno, estão concentrados entre a região metropolitana (1), a serra (2) e o norte (5). A frequência relativa de SRAG é maior nos municípios com baixo IPS. Nos cortes de classe definidos como IPS "baixo" (B) e "muito baixo" (MB), a média foi de $25,7 \%$ dos óbitos. Por outro lado, a frequência relativa média nos cortes de classe definidos como "alto" (B) e "muito alto" (MA) foi de 13,6\%. A frequência relativa do ano de 2020 não difere muito dos anos anteriores, sendo maior nos municípios com baixo IPS e menor nos municípios com elevado IPS. 
A taxa de mortalidade foi também maior nos municípios com baixo IPS; apenas o ano de 2017 apresentou valores parecidos entre municípios de maior e menor privação. As taxas são maiores no ano de 2020 em todos os níveis de privação, mas as mais elevadas também foram encontradas nos municípios de menor IPS, como vinha ocorrendo nos anos anteriores. A distribuição espacial e a taxa de mortalidade por COVID-19 acompanham a mesma geografia da mortalidade por SRAG, sendo mais elevada nos municípios do eixo capital-serra-norte do estado, com menor privação social, e menor nos municípios ao sul e oeste, onde estão os municípios de maior privação social.

\section{DISCUSSÃO}

O aumento de óbitos por SRAG nos meses de março a junho de 2020, acompanhado da sua variação demográfica diferente em relação aos anos anteriores, é condizente com a hipótese de que óbitos por COVID-19 estejam sendo registrado no sistema de vigilância da SRAG (BASTOS et al., 2020). O aumento da frequência relativa de casos entre idosos e a redução da frequência relativa na população de jovens e crianças, acompanhada do aumento proporcional muito maior da taxa de mortalidade entre idosos em 2020, assinala a necessidade do aumento de testes para comprovação do agente etiológico dos óbitos. O elevado número de óbitos por SRAG não especificados etiologicamente pode ser resultado do aumento de testes laboratoriais negativados (MINISTÉRIO DA SAÚDE BRASIL, 2020; BASTOS et al., 2020). Por outro lado, o fato de 2020 ser o ano com o menor número de óbitos por influenza, parece refletir a antecipação da campanha de vacinação em todo o Brasil. Isso reforça ainda mais a existência de um novo vírus que está circulando e que, possivelmente, não esteja sendo adequadamente avaliado pelo painel de controle da SRAG. É recomendado, dessa forma, ampliar a cobertura de testes para COVID-19 nos óbitos com resultados laboratoriais negativados para influenza e outros vírus respiratórios.

O aumento da mortalidade por SRAG em 2020 é ainda mais preocupante devido as profundas desigualdades sociais do Rio Grande do Sul, como no Brasil, em geral. A SRAG mata mais a população negra e indígena, como também a população com baixa escolaridade em toda a série histórica. Portanto, são diferentes as possibilidades de sobrevida, que vão depender das condições prévias e gerais de vida e saúde, como também da maior ou menor exposição ao vírus. O que difere o ano 2020 em relação aos demais é o aumento absurdo das taxas em todos os níveis escolares e em todas as classificações raciais. Pelos estudos que vem sendo apresentados sobre o impacto da COVID-19 na população negra e indígena (GOES; RAMOS; FERREIRA, 2020; BAQUI et al.; PRICE-HAYWOOD et al., 2020; CUPERTINO et al., 2020), é recomendado enorme atenção aos impactos socialmente seletivos da epidemia. Uma das ações necessárias, para além da ampliação dos sistemas de proteção, é ainda o aumento da cobertura de testes para garantir o controle da difusão do vírus nas populações socialmente mais carenciadas. Deve ser referido a precariedade dos dados para a determinação das 
desigualdades sociais na mortalidade por SRAG e COVID-19 no Brasil, embora seja amplamente reconhecida a determinação social para esses indicadores (ABRAMS; SZEFLER, 2020; BAMBRA et al., 2020; TAKIAN; KIANI; KHATERE KHANJANKHANI, 2020).

A distribuição espacial dos óbitos por SRAG e por COVID-19 é condizente com a formação social e territorial do Rio Grande do Sul. Em primeiro lugar, síndromes respiratórias são, em geral, de difícil controle espacial, senão apenas com medidas de contenção espacial rígidas, como o lockdown, por exemplo (MORITZ, 2020). Como a transmissão se dá pelo contato com pessoas ou com superfícies infectadas, os vírus causadores das SRAG avançam sempre dos municípios de maior adensamento e de maior fluxo humano, para os municípios remotos de menor porte, sob o padrão claramente hierárquico (HAGGETT, 2000). Por essa razão, é maior o número de casos entre a região metropolitana de Porto Alegre, Caxias do Sul e Passo Fundo, no eixo assim chamado capital-serra-norte, que é o mais desenvolvido, mais denso e de maior fluidez populacional (LAZARETTI; TEIXEIRA, 2019). Com pequenas variações, esse foi o padrão espacial encontrado para todos os anos avaliados, com diferença singular em 2020 que é a extensão geográfica do evento, presente em praticamente todas as regiões do estado.

Excetuando-se o ano de 2016 e 2020, o primeiro marcado pela maior epidemia de influenza desde 2009 (CEVS, 2020), os demais apresentaram uma distribuição espacial discreta, relativamente confinada à região metropolitana. A distribuição geográfica dos óbitos por SRAG em 2020 segue o mesmo padrão da distribuição espacial dos casos de COVID-19 no Rio Grande do Sul (FARIA et al., 2020). De tal forma, no eixo, capital-serra-norte estão concentrados mais de $80 \%$ dos óbitos por SRAG, acompanhado de outros eixos secundários em direção ao centro e sul do estado (sobretudo nas regiões de Santa Maria, Pelotas e Bagé).

O Rio Grande do Sul é reconhecido pelas profundas desigualdades sociais entre as regiões norte e sul do estado, frequentemente simbolizadas pelas disparidades das condições de vida entre a Serra e o Pampa (LAZARETTI; TEIXEIRA, 2019). Os resultados deste estudo não mostraram desigualdades territoriais nas taxas de mortalidade por SRAG, pelo menos no intervalo temporal avaliado. É maior a taxa de mortalidade nos municípios com menor privação social do eixo capital-serra-norte, e menor a taxa de mortalidade nos municípios de maior privação, notadamente na região do Pampa. Em termos de escala, pode-se dizer que existem profundas desigualdades sociais (raça e escolaridade, por exemplo), possivelmente mais densas nas periferias das cidades, sobretudo nas grandes cidades. Mas na escala regional do estado, todavia, os resultados ainda não permitem determinar essas desigualdades na taxa de mortalidade por SRAG e por COVID-19. Pode-se sugerir que a taxa de mortalidade é maior nos municípios de menor privação exatamente porque eles são mais acometidos, uma vez que a propagação é maior nas áreas de maior fluxo da rede urbana (MORITZ, 2020) (DANON et al., 2020). Por outro lado, os vazios assistenciais nas áreas de maior privação social, sobretudo nos 
municípios de enormes dimensões territoriais do interior e das fronteiras, talvez sejam determinantes para subnotificação dos casos. Por isso, é necessário ampliar o sistema de atenção e vigilância à SRAG e à COVID-19 nestas áreas remotas, que é a única forma de se ter a real dimensão da epidemia no estado.

A extensão geográfica dos óbitos por SRAG em 2020, presente em mais da metade dos municípios do estado do Rio Grande do Sul, indica também a interiorização dos casos. Isso traz ainda uma dificuldade, que é a vigilância e planejamento territorial dos serviços e dos sistemas logísticos que conectam municípios de enorme extensão territorial e de baixa densidade demográfica. O Pampa, em geral, e a fronteira sul e oeste, em particular, precisam ser pensadas pelas características que lhes são próprias. Daí a importância de se propor ações específicas para o eixo que vai de Pelotas e Bagé até Uruguaiana, atravessando toda fronteira com Uruguai, de um lado, e a região central, que vai de Santa Maria à Santa Rosa, estendendo-se até a fronteira com a Argentina. Recomenda-se, entre outros, aumentar a capacidade de atendimento e também o sistema logístico necessário para transporte de pacientes em longas distâncias.

Outro elemento importante que torna os dados de 2020 preocupantes é o fato de se tratar ainda de pré-crise para SRAG. É sabido que o Rio Grande do Sul tem o pico dos casos no inverno, sobretudo nos meses de julho e agosto (FIOCRUZ, 2020; CEVS, 2020). Portanto, os dados de março e junho assinalam um cenário de crise que precisa ser avaliada cuidadosamente pela vigilância epidemiológica, sob o risco de se ter uma explosão de internações e óbitos por SRAG num momento que, pelo que se demostra nas avaliações estatísticas da COVID-19 para o Rio Grande do Sul, o estado está em processo de crescimento das taxas e com relativa imprevisibilidade para se determinar o esperado platô (HALLAL et al., 2020; FARIA et al., 2020).

Finalmente, os resultados da variação demográfica indicam um cenário que deve ser cuidadosamente avaliado. O Rio Grande do Sul possui, proporcionalmente, a maior população idosa do país, na ordem de 18,2\% de acordo com estimativa do IBGE (2019), a média brasileira é de 13,8\%. A elevada taxa de mortalidade por SRAG na população idosa no ano de 2020 (81,1 por 100 mil/ habitantes, enquanto a média do estado foi de 18,1 no mesmo período), é algo anômalo e deve ser considerado como uma excepcionalidade. Esse cenário sugere enormes cuidados e políticas de prevenção à difusão da SRAG, em geral, e da COVID-19, em particular, dado a letalidade muito maior nesse grupo populacional. Isso coloca também o Rio Grande do Sul numa situação ímpar em relação ao restante do país, inclusive aos seus vizinhos regionais, que possuem um índice de envelhecimento bem menor que o gaúcho. Num momento de aumento da mortalidade por COVID-19 no estado, acompanhada da sua interiorização, é recomendado, portanto, aprofundar as políticas de prevenção, sobretudo as de isolamento social, criar políticas de apoio às populações em condições em situação de risco e de maior vulnerabilidade, e aumentar a cobertura dos serviços de saúde para uma demanda cada vez mais crescente. 


\section{CONSIDERAÇÕES FINAIS}

O aumento da mortalidade por SRAG no Rio Grande do Sul, as variações demográficas e a ausência da identificação do agente etiológico são possíveis resultados da difusão da COVID-19 no estado. Este estudo sugere a necessidade urgente de ampliar a cobertura de exames de COVID-19 na população cujo óbito foi associado à SRAG. É preciso ainda associar a ampliação dos exames ao desenvolvimento de políticas imediatas com foco em grupos populacionais mais vulneráveis, nomeadamente os idosos, os negros e os indígenas.

No ano de 2020 a mortalidade por SR AG generalizou-se para quase todas as regiões do estado, em quantidade e com taxas muito superiores à série histórica. A antecipação da campanha de vacinação contra gripe foi importante e resultou numa menor mortalidade pelo vírus influenza. Mas não foi capaz de conter o aumento da mortalidade por SRAG, provocada pela epidemia de COVID-19, tanto pelos casos etiologicamente assim classificados, como também pelos casos possivelmente subnotificados. Isso assinala um enorme problema para um estado com inverno rigoroso e uma população relativamente idosa. É de se esperar que nos três próximos meses, salvo medidas imediatas, esses casos irão aumentar e se estender geograficamente.

O estudo mostrou também que o eixo principal de densificação dos óbitos ocorre na área de menor privação social do estado (capital-serra-norte). As razões podem estar associadas à própria dinâmica da difusão das síndromes respiratórias, que é a maior nas áreas de maior densidade e fluxo populacional. Entretanto, o registro de casos em mais da metade dos municípios gaúchos denota uma preocupação com os municípios de elevada privação social localizados, sobretudo, próximos das fronteiras sul e oeste. Por isso, é importante dimensionar os impactos nesses locais de maior privação social, criando-se estruturas de resposta em locais de difícil acesso e de menor densidade de serviços de saúde.

\section{AGRADECIMENTOS}

O presente trabalho foi realizado com apoio da Coordenação de Aperfeiçoamento de Pessoal de Nível Superior - Brasil (CAPES) - Código de Financiamento 001 e ao Programa Nacional de Pós-Doutorado (PNPD -CAPES) - e ao Observatório de Informações em Saúde da Universidade Federal de Santa Maria.

\section{REFERÊNCIAS}

ABRAMS, E. M.; SZEFLER, S. J. COVID-19 and the impact of social determinants of health. The Lancet Respiratory Medicine, v. 8, n. 7, p. 659- 661, 2020. 
ALONSO, W. J. et al. Covid-19 em contexto: comparação com a mortalidade mensal por causas respiratórias nos estados brasileiros. Interamerican Journal of Medicine and Health, v. 3, 2020.

BAMBRA, C. et al. The COVID-19 pandemic and health inequalities. Journal of Epidemiology and Community Health, p. 1-5, 2020.

BAQUI, P. O. et al. Ethnic and regional variation in hospital mortality from COVID-19 in Brazil. The Lancet Global Health, p. 1-9, 2020.

BASTOS, L. S. et al. COVID-19 e hospitalizações por SRAG no Brasil: uma comparação até a 12a semana epidemiológica de 2020. Cadernos de Saúde Pública, v. 36, n. 4, 2020.

CÂMARA, G. et al. Análise de dados de área. CÂMARA, G. et al. In: Análise Espacial de Dados Geográficos. Brasília: EMBRAPA, 2004.

CAO, Z. et al. Estimating the effective reproduction number of the 2019-nCoV in China. medRxiv, 2020.

CARSTAIRS, V.; MORRIS, R. Deprivation and health in Scotland. Health bulletin, v. 48, n. 4, p. 162-175, 1990.

CENTRO ESTADUAL DE VIGILANCIA EM SAÚDE (CEVS). Situação Epidemiológica/Dados. 2020. Disponível em: https://bit.ly/2IktI2q. Acesso em 10 de jul. 2020.

CHEN, S. et al. Mitigating COVID-19 outbreak via high testing capacity and strong transmission-intervention in the United States. medRxiv, 2020.

CUPERTINO, G. A. et al. COVID-19 and Brazilian Indigenous Populations. The American Journal of Tropical Medicine and Hygiene, p. 1-4, 2020.

DANON, L. et al. A spatial model of CoVID-19 transmission in England and Wales: early spread and peak timing. medRxiv, 2020.

FARIA, R. et al. Difusão da covid-19 nas grandes estruturas territoriais do estado do Rio Grande do Sul, Brasil. Hygeia - Revista Brasileira de Geografia Médica e da Saúde, v. 17, p. 1-10, 2020. 
FUNDAÇÃO OSWALDO CRUZ (FIOCRUZ). Monitoramento de casos reportados de síndrome respiratória aguda grave (SRAG) hospitalizados. 2020. Disponível em: http://info.gripe.fiocruz.br/. Acesso em 21 de jul. 2020.

GOES, E. F.; RAMOS, D. DE O.; FERREIRA, A. J. F. Desigualdades raciais em saúde e a pandemia da Covid-19. Trabalho, Educação e Saúde, v. 18, n. 3, p. 1-7, 2020.

HAGGETT, P. The geographical structure of epidemics. Oxford University Press, 2000.

HALLAL, P. C. et al. Evolução da prevalência de infecção por COVID-19 no Rio Grande do Sul: inquéritos sorológicos seriados. Ciência da Saúde Coletiva, 2020.

INSTITUTO BRASILEIRO DE GEOGRAFIA E ESTATÍSTICA (IBGE). Estimativas da população. 2019. Disponível em: https://bit.ly/32pS1TU. Acesso em: 30 de mar. 2020.

LANA, R. M. et al. Emergência do novo coronavírus (SARS-CoV-2) e o papel de uma vigilância nacional em saúde oportuna e efetiva. Cadernos de Saúde Pública, v. 36, n. 3, 2020.

LAZARETTI, L. R.; TEIXEIRA, F. O. Desigualdades socioeconômicas e demográficas nos municípios do Rio Grande do Sul/Brasil: um índice de vulnerabilidade à pobreza. URBE - Revista Brasileira de Gestão Urbana, v. 11, p. 1-15, 2019.

LEE, T. H. et al. Testing for SARS-CoV-2: Can We Stop at Two? Clinical infectious diseases: an official publication of the Infectious Diseases Society of America, 2020.

LISBOA, L. et al. Mortalidade infantil: principais causas evitáveis na região Centro de Minas Gerais, 1999-2011. Epidemiologia e Serviços de Saúde, v. 24, n. 4, p. 711-720, 2015.

LIU, T. et al. Transmission Dynamics of 2019 Novel Coronavirus (2019-nCoV). SSRN Electronic Journal, 2020.

LOBO, A. P. et al. COVID-19 epidemic in Brazil: Where are we at? International Journal of Infectious Diseases, v. 97, p. 382-385, 2020.

MARCEL, S. et al. COVID-19 epidemic in Switzerland: On the importance of testing, contact tracing and isolation. Swiss Medical Weekly, v. 150, n. 11-12, 2020. 
MINISTÉRIO DA SAÚDE BRASIL. Boletim Epidemiológico Especial COE-COVID-19. Brasília, 2020. Disponível em: https://www.saude.gov.br/. Acesso em 19 de jul. 2020.

MIZUMOTO, K. Estimating the asymptomatic proportion of coronavirus disease 2019 (COVID-19) cases on board the Diamond Princess cruise ship, Yokohama, Japan, 2020.

MORITZ, U. Et al. The effect of human mobility and control measures on the COVID-19 epidemic in China. Science, v. 368, n. 6490, p. 493-497, 2020.

PRADO, M. et al. Nota Técnica 7 - 11/04/2020 Análise de subnotificação do número de casos confirmados da COVID-19 no Brasil. Rio de Janeiro, 2020. Disponível em: https://bit.ly/35hmgyt. Acesso em 19 de jul. 2020.

PRICE-HAYWOOD, E. G. et al. Hospitalization and Mortality among Black Patients and White Patients with Covid-19. New England Journal of Medicine, v. 382, n. 26, p. 1-10, 2020.

PROGRAMA DAS NAÇÕES UNIDAS PARA O DESENVOLVIMENTO (PNUD). Atlas do Desenvolvimento Humano no Brasil. Disponível em: http://atlasbrasil.org.br/2013/. Acesso em: 5 de mai. 2020.

RIZZATTI, M. et al. Mapeamento da COVID-19 por meio da densidade de Kernel. Metodologias e Aprendizado, p. 44-53, 2020.

SAATY, T. L. How to make a decision: The analytic hierarchy process. European Journal of Operational Research, v. 48, n. 1, p. 9-26, 1990.

TAKIAN, A.; MEHDI KIANI, M.; KHANJANKHANI, K. COVID-19 and the need to prioritize health equity and social determinants of health. International Journal of Public Health, 2020.

UNIVERSIDADE FEDERAL DE PELOTAS (UFPEL). EPICOVID19-BR relatório 02 de julho de 2020. 2020. Disponível em: https://bit.ly/2Ij48LM. Acesso em: 5 de jul. 2020. 
